\title{
A prática profissional em Centro de Atenção Psicossocial: A produção científica na
}

\section{pós-graduação}

\author{
Professional practice in a Psychosocial Care Center: Scientific production in graduate studies \\ Práctica profesional en un Centro de Atención Psicosocial: Producción científica en estudios de \\ posgrado
}

Recebido: 23/03/2021 | Revisado: 01/04/2021 | Aceito: 03/04/2021 | Publicado: 13/04/2021

\author{
Rafael Pasche da Silveira \\ ORCID: https://orcid.org/0000-0003-4695-1381 \\ Universidade Federal de Santa Maria, Brasil \\ E-mail: rafaelpasche@gmail.com \\ Rosangela Marion da Silva \\ ORCID: https://orcid.org/0000-0003-3978-9654 \\ Universidade Federal de Santa Maria, Brasil \\ E-mail: cucasma@terra.com.br \\ Fernanda Demetrio Wasum \\ ORCID: https://orcid.org/0000-0002-3053-4965 \\ Universidade Federal de Santa Maria, Brasil \\ E-mail: fernandawasum@gmail.com \\ Larissa Dias Antunes \\ ORCID: https://orcid.org/0000-0002-5852-4910 \\ Universidade Federal de Santa Maria, Brasil \\ E-mail: larissadiasanttunes@gmail.com \\ Daiana Foggiato de Siqueira \\ ORCID: https://orcid.org/0000-0002-8592-379X \\ Universidade Federal de Santa Maria, Brasil \\ E-mail: daianasiqueira@yahoo.com.br
}

\begin{abstract}
Resumo
Objetivo: $\mathrm{O}$ estudo tem por objetivo conhecer e analisar a tendência das produções científicas brasileiras sobre a prática profissional em Centros de Atenção Psicossocial (CAPS). Método: Trata-se de uma revisão narrativa da literatura, realizada no portal de teses e dissertações da Capes, no mês de julho de 2020. As palavras utilizadas na busca foram: [profissona*] AND [prática] AND [CAPS] OR [centro de atenção psicossocial], empregando operador booleano "AND" ou "OR" entre elas. Resultados: Foram encontradas 217 teses e dissertações. A partir do resultado desta busca foi realizada a leitura dos resumos e após aplicação de critérios de inclusão e exclusão, restaram 18 dissertações e 2 teses. A análise das 20 produções, na íntegra, se deu por meio da análise de conteúdo de Bardin. As temáticas que emergiram a partir das produções estabelecidas foram: $\mathrm{O}$ cuidado em saúde mental e a prática profissional na assistência em saúde mental. Conclusão: Conclui-se que a abordagem qualitativa é frequente em teses e dissertações sobre o tema e que são necessárias pesquisas que qualifiquem a prática dos profissionais dentro dos CAPS e na saúde mental de modo geral.
\end{abstract}

Palavras-chave: Pessoal de saúde; Saúde mental; Educação de pós-graduação.

\begin{abstract}
Objective: The study aims to understand and analyze the trend of Brazilian scientific productions on professional practice in Psychosocial Care Centers (CAPS). Method: This is a narrative review of the literature, carried out on Capes' theses and dissertations portal, in July 2020. The words used in the search were: [profissona *] AND [practical] AND [CAPS] OR [ psychosocial care center], employing a Boolean operator "AND" or "OR" between them. Results: 217 theses and dissertations were found. From the result of this search, the abstracts were read and after applying inclusion and exclusion criteria, 18 dissertations and 2 theses remained. The analysis of the 20 productions, in full, took place through Bardin's content analysis. The themes that emerged from the established productions were: Mental health care and professional practice in mental health care. Conclusion: It is concluded that the qualitative approach is frequent in theses and dissertations on the theme and that research is needed to qualify the practice of professionals within the CAPS and in mental health in general.
\end{abstract}

Keywords: Health personnel; Mental health; Education Graduate. 


\begin{abstract}
Resumen
Objetivo: El estudio tiene como objetivo comprender y analizar la tendencia de las producciones científicas brasileñas sobre la práctica profesional en los Centros de Atención Psicosocial (CAPS). Método: Se trata de una revisión narrativa de la literatura, realizada en el portal de tesis y disertaciones de Capes, en julio de 2020. Las palabras utilizadas en la búsqueda fueron: [profissona *] Y [práctica] Y [CAPS] O [centro de atención psicosocial ], empleando un operador booleano "Y" u "O" entre ellos. Resultados: se encontraron 217 tesis y disertaciones. A partir del resultado de esta búsqueda se leyeron los resúmenes y luego de aplicar criterios de inclusión y exclusión quedaron 18 disertaciones y 2 tesis. El análisis de las 20 producciones, en su totalidad, se realizó a través del análisis de contenido de Bardin. Los temas que surgieron de las producciones establecidas fueron: Cuidado de la salud mental y práctica profesional en el cuidado de la salud mental. Conclusión: Se concluye que el abordaje cualitativo es frecuente en tesis y disertaciones sobre el tema y que se necesita investigación para calificar la práctica de los profesionales dentro del CAPS y en salud mental en general.
\end{abstract}

Palabras clave: Personal de salud; Salud mental; Educación de posgrado.

\title{
1. Introdução
}

Pensar em pesquisar temas que envolvam a saúde mental remete a entender como se vive o momento atual de cuidado. A história da loucura por meio do tempo mostrou a pessoa com transtorno mental como um sujeito a margem da sociedade, nesse contexto havia preconceito, exclusão e até mesmo um tratamento violento quando este necessitava de assistência em saúde (Foucault, 2019).

A política de saúde Mental brasileira é reconhecida como referência em um processo de superação de uma lógica de cuidado manicomial, tendo a Reforma Psiquiátrica brasileira como início da busca por serviços de cuidado para transtornos mentais e transtornos relacionados ao uso de álcool e outras drogas em um contexto extra-hospitalar (Carvalho et al, 2017).

$\mathrm{O}$ tratamento em saúde mental teve sua mudança consolidada pela lei $\mathrm{N}^{\circ} 10.216$, conhecida também como lei Paulo Delgado, instituindo um novo modelo de cuidado para pessoas com transtornos mentais, trazendo um direcionamento ao cuidado em serviços de base comunitária e de respeito aos seus direitos (Brasil, 2001). Esta lei é um marco na história do Brasil no que diz respeito a saúde mental, contudo pode-se ter uma crítica por ela não deixar claro sobre como acontece a redução de leitos manicomiais.

$\mathrm{Na}$ busca pela construção do cuidado com lógica comunitária foi estabelecida a portaria $\mathrm{N}^{\circ} 336 \mathrm{em} 2002$. Este documento trata do funcionamento dos Centros de Atenção Psicossocial (CAPS), trazendo a constituição da equipe e suas modalidades conforme especificidade do público e população do município (Brasil, 2002). Cabe ressaltar que o primeiro serviço na modalidade CAPS foi instituído no Brasil em 1986 (Ferreira et al, 2016), assim a lei veio a consolidar um modelo já existente.

Desta forma, no caminho por melhoras na assistência em saúde mental, em 2011 foi Instituída a Rede de Atenção Psicossocial (RAPS) para pessoas com sofrimento ou transtorno mental e com necessidades decorrentes do uso de álcool e outras drogas, no âmbito do Sistema Único de Saúde (SUS), com a finalidade de criação, ampliação e articulação de pontos de atenção à saúde dessa população (Brasil, 2011).

Colocando o CAPS como serviço essencial da RAPS é necessário compreender o cuidado prestado nesse serviço. A equipe de assistência é fundamental para motivar o tratamento dos usuários acompanhados em um CAPS, usuários relatam essa motivação a partir da relação que estabelecem com quem presta seu cuidado (Santos et al, 2018). Assim, é importante que os profissionais dos serviços estejam atuando de maneira coerente com as políticas de saúde mental e também em plenas condições de saúde.

No Brasil é observada uma deterioração nas condições de trabalho de profissionais da saúde, sendo um reflexo também de questões políticas que envolvem a organização do trabalho, as quais refletem em falta de recursos materiais, profissionais em número inadequado, qualificações deficitárias e baixos salários. Estes fatores trazem sofrimento aos profissionais, por impactos em valores sociais, organizacionais e políticos de categorias trabalhistas (Dias et al, 2019). 
Pensar na questão da prática profissional remete a perceber a subjetividade dos sujeitos envolvidos em tal processo (Souza \& Bernardo, 2019). Desta forma é necessário entender a percepção dos trabalhadores sobre as experiências que este vivencia e como percebe suas práticas dentro do contexto do cuidado aos usuários.

A partir do exposto, questionou-se qual a tendência das produções científicas da pós-graduação brasileira sobre a prática profissional em Centros de Atenção Psicossocial? Partindo desse questionamento, o presente estudo objetiva conhecer e analisar a tendência das produções científicas brasileiras sobre a prática profissional em Centros de Atenção Psicossocial.

\section{Metodologia}

Esta pesquisa trata-se de uma revisão narrativa da literatura, realizada por meio do portal de teses e dissertação da Capes no mês de julho de 2020. A busca foi realizada por meio da inserção de termos (palavras) na caixa de busca do portal. As palavras utilizadas na busca foram: [profissiona*] AND [prática] e [CAPS] OR [centro de atenção psicossocial], empregando operador booleando "AND" e "OR" entre elas. Foram encontradas 217 teses e dissertações. Como critérios de inclusão foram escolhidas as teses e dissertações que fossem pertinentes à temática em estudo e respondessem a pergunta e como critérios de exclusão estudos sobre práticas especificas (grupos, oficinas, abordagens especificas, escutas qualificadas, atividades focadas ou modelos de abordagens), abordagens de núcleos específicos de profissionais ou não ter acesso ao resumo de maneira online e gratuita. A partir da aplicação dos critérios de inclusão, restaram 18 dissertações e 2 teses. O Quadro 1 apresenta as produções selecionadas no estudo.

Quadro 1. Relação de Teses e Dissertações que foram selecionadas no banco de teses e dissertações da CAPES após aplicação de critérios de inclusão e exclusão (continuação).

\begin{tabular}{|c|c|c|c|c|}
\hline $\begin{array}{l}\text { Código/ } \\
\text { Autor }\end{array}$ & Inst.* & Título & Tipo/ano & Programa \\
\hline $\begin{array}{l}\text { E1 } \\
\text { Duarte, Mara Cristina } \\
\text { Ribeiro }\end{array}$ & USP & $\begin{array}{l}\text { A reabilitação psicossocial num CAPS: concepção } \\
\text { dos profissionais }\end{array}$ & $\begin{array}{l}\text { Mestrado } \\
\text { Acadêmico/2005 }\end{array}$ & Enfermagem \\
\hline $\begin{array}{l}\text { E2 } \\
\text { Bichaff, Regina }\end{array}$ & USP & $\begin{array}{l}\text { O trabalho nos Centros de Atenção Psicossocial: } \\
\text { uma reflexão crítica das práticas e suas } \\
\text { contribuições para a consolidação da reforma } \\
\text { psiquiátrica }\end{array}$ & $\begin{array}{l}\text { Mestrado } \\
\text { Acadêmico/2006 }\end{array}$ & Enfermagem \\
\hline $\begin{array}{l}\text { E3 } \\
\text { Santos, Gláucia } \\
\text { Lorena Guedes dos. }\end{array}$ & UFPE & $\begin{array}{l}\text { Sobre discursos e práticas: a reabilitação } \\
\text { psicossocial pelo "olhar" dos técnicos de referência } \\
\text { de um CAPS da região metropolitana de recife }\end{array}$ & $\begin{array}{l}\text { Mestrado } \\
\text { Acadêmico/2008 }\end{array}$ & Psicologia \\
\hline $\begin{array}{l}\text { E4 } \\
\text { Dos Santos, Jamerson } \\
\text { Luis Gonçalves }\end{array}$ & UCSAL & $\begin{array}{l}\text { Política de saúde pública para usuários de álcool e } \\
\text { outras drogas no brasil: a prática no CAPS ad em } \\
\text { feira de Santana, Bahia, brasil }\end{array}$ & $\begin{array}{l}\text { Mestrado } \\
\text { Acadêmico/2009 }\end{array}$ & $\begin{array}{l}\text { Políticas sociais e } \\
\text { cidadania }\end{array}$ \\
\hline $\begin{array}{l}\text { E5 } \\
\text { Lúcia Dutra } \\
\text { Facundes, Vera }\end{array}$ & UFPE & $\begin{array}{l}\text { O discurso e a prática no Centro de Atenção } \\
\text { Psicossocial: a construção do cuidado em saúde } \\
\text { mental }\end{array}$ & Doutorado/2010 & Neuropsiquiatria \\
\hline $\begin{array}{l}\text { E6 } \\
\text { Marinho, Angélica } \\
\text { Mota }\end{array}$ & UFCE & $\begin{array}{l}\text { Centro de Atenção Psicossocial álcool e outras } \\
\text { drogas: re-construção de uma prática }\end{array}$ & $\begin{array}{l}\text { Mestrado } \\
\text { Acadêmico/2010 }\end{array}$ & Enfermagem \\
\hline $\begin{array}{l}\text { E7 } \\
\text { Antonio, Priscila da } \\
\text { Silva }\end{array}$ & UNB & $\begin{array}{l}\text { O cotidiano do CAPS: investigação do amar, gozar, } \\
\text { trabalhar e comunicar em profissionais de saúde } \\
\text { mental }\end{array}$ & $\begin{array}{l}\text { Mestrado } \\
\text { Acadêmico/2011 }\end{array}$ & Psicologia \\
\hline $\begin{array}{l}\text { E8 } \\
\text { Espinola, } \\
\text { Lawrencita Limeira }\end{array}$ & UFPB & $\begin{array}{l}\text { Práticas do cuidar de adolescentes usuários de } \\
\text { drogas }\end{array}$ & $\begin{array}{l}\text { Mestrado } \\
\text { Acadêmico/2013 }\end{array}$ & Enfermagem \\
\hline $\begin{array}{l}\text { E9 } \\
\text { Nascimento, Renata } \\
\text { Maria da Silva }\end{array}$ & $\begin{array}{l}\text { Fiocruz- } \\
\text { PE }\end{array}$ & $\begin{array}{l}\text { A integralidade na política de saúde mental: análise } \\
\text { da prática e do discurso em um Centro de Atenção } \\
\text { Psicossocial }\end{array}$ & $\begin{array}{l}\text { Mestrado } \\
\text { Acadêmico/2014 }\end{array}$ & Saúde pública \\
\hline
\end{tabular}


Research, Society and Development, v. 10, n. 4, e35010414190, 2021

(CC BY 4.0) | ISSN 2525-3409 | DOI: http://dx.doi.org/10.33448/rsd-v10i4.14190

\begin{tabular}{|c|c|c|c|c|}
\hline $\begin{array}{l}\text { E10 } \\
\text { Mochel, José } \\
\text { Augusto Gomide }\end{array}$ & UFMA & $\begin{array}{l}\text { A clínica da atenção psicossocial e a dependência de } \\
\text { drogas: análise dos discursos de profissionais e de } \\
\text { sujeitos em tratamento no CAPS ad }\end{array}$ & $\begin{array}{l}\text { Mestrado } \\
\text { Acadêmico/2014 }\end{array}$ & Psicologia \\
\hline $\begin{array}{l}\text { E11 } \\
\text { Bettin, Andréia } \\
\text { Coelho }\end{array}$ & UFPEL & $\begin{array}{l}\text { Processos inter-relacionais nos CAPS: o desafio } \\
\text { para construção de equipes interdisciplinares com } \\
\text { vínculos profissionais saudáveis }\end{array}$ & $\begin{array}{l}\text { Mestrado } \\
\text { Acadêmico/2014 }\end{array}$ & Enfermagem \\
\hline $\begin{array}{l}\text { E12 } \\
\text { Da Silva, } \\
\text { Enio Rodrigues }\end{array}$ & UFMG & $\begin{array}{l}\text { O gesto profissional em psiquiatria; o Centro de } \\
\text { Atenção Psicossocial como território de trabalho }\end{array}$ & Doutorado/2016 & Educação \\
\hline $\begin{array}{l}\text { E13 } \\
\text { Silveira, Paula } \\
\text { Morena Souto } \\
\text { Derenusson }\end{array}$ & USP & $\begin{array}{l}\text { Saúde mental: concepções e práticas profissionais de } \\
\text { um campo em tensão }\end{array}$ & $\begin{array}{l}\text { Mestrado } \\
\text { Acadêmico/2016 }\end{array}$ & Saúde pública \\
\hline $\begin{array}{l}\text { E14 } \\
\text { Adachi, Flavia } \\
\text { Vernizi }\end{array}$ & UTP & $\begin{array}{l}\text { Avaliação da prática desenvolvida num centro de } \\
\text { atenção psicossocial - CAPSIII do município de } \\
\text { Curitiba }\end{array}$ & $\begin{array}{l}\text { Mestrado } \\
\text { Acadêmico/2017 }\end{array}$ & Psicologia \\
\hline $\begin{array}{l}\text { E15 } \\
\text { Santos, Sueli de } \\
\text { Farias }\end{array}$ & $\begin{array}{l}\text { UNIOEST } \\
\text { E }\end{array}$ & $\begin{array}{l}\text { Organização do trabalho de atendimento a usuários } \\
\text { com transtorno mental severo e persistente no } \\
\text { Centro de Atenção Psicossocial II em foz do Iguaçu }\end{array}$ & $\begin{array}{l}\text { Mestrado } \\
\text { Acadêmico/2017 }\end{array}$ & $\begin{array}{l}\text { Sociedade, cultura e } \\
\text { fronteiras }\end{array}$ \\
\hline $\begin{array}{l}\text { E16 } \\
\text { Wagner, Natalia } \\
\text { Cyrillo }\end{array}$ & UNIFESP & $\begin{array}{l}\text { Desenvolvimento do cuidado em saúde mental no } \\
\text { contexto de consolidação da reforma psiquiátrica: a } \\
\text { experiência de um CAPS em santos }\end{array}$ & $\begin{array}{l}\text { Mestrado } \\
\text { profissional/2017 }\end{array}$ & Ciências da saúde \\
\hline $\begin{array}{l}\text { E17 } \\
\text { Barroso, Claudenia } \\
\text { Mesquita }\end{array}$ & UFCE & $\begin{array}{l}\text { Navegar é preciso" - cartografando subjetividades } \\
\text { em saúde mental }\end{array}$ & $\begin{array}{l}\text { Mestrado } \\
\text { Acadêmico/2017 }\end{array}$ & Saúde da família \\
\hline $\begin{array}{l}\text { E18 } \\
\text { Guimaraes, Lislaine } \\
\text { Santos }\end{array}$ & UNIFESP & $\begin{array}{l}\text { Concepções e práticas de cuidado em saúde mental } \\
\text { a partir de diferentes formações profissionais }\end{array}$ & $\begin{array}{l}\text { Mestrado } \\
\text { profissional/2017 }\end{array}$ & Ciências da saúde \\
\hline $\begin{array}{l}\text { E19 } \\
\text { Moraes, Peggy Liz } \\
\text { Mendes de }\end{array}$ & UFF & $\begin{array}{l}\text { Percepção fenomenológica dos profissionais do } \\
\text { Centro de Atenção Psicossocial (CAPS): vivências e } \\
\text { desafios da prática em saúde mental }\end{array}$ & $\begin{array}{l}\text { Mestrado } \\
\text { Acadêmico/2018 }\end{array}$ & Enfermagem \\
\hline $\begin{array}{l}\text { E20 } \\
\text { Freitas, Valeria } \\
\text { Nancy De }\end{array}$ & UNIFESP & $\begin{array}{l}\text { Reabilitação psicossocial: concepções e práticas na } \\
\text { construção de uma rede possível }\end{array}$ & $\begin{array}{l}\text { Mestrado } \\
\text { Profissional/2018 }\end{array}$ & Ciência da saúde \\
\hline
\end{tabular}

*Int.= instituição. Fonte: Autores.

Para organizar os dados foi utilizado um quadro sinóptico analítico e seu conteúdo foi analisado conforme proposta de Bardin (2011). Ressalta-se que os aspectos éticos foram respeitados, conforme a Lei de Direitos Autorais $\mathrm{n}^{\circ} .9 .610$ de 19 de fevereiro de 1998.

\section{Resultados}

Após aplicar os critérios de inclusão e exclusão, foram selecionados 20 estudos, sendo 18 dissertações (90\%) e 2 teses (10\%). Organizadas conforme ano de produção constitui-se a Tabela 2. 
Tabela 1. Distribuição dos estudos conforme ano de defesa.

\begin{tabular}{cc}
\hline Ano & Frequência (\%) \\
\hline 2005 & $01(5 \%)$ \\
2006 & $01(5 \%)$ \\
2008 & $01(5 \%)$ \\
2009 & $01(5 \%)$ \\
2010 & $02(10 \%)$ \\
2011 & $02(10 \%)$ \\
2013 & $01(5 \%)$ \\
2014 & $03(15 \%)$ \\
2016 & $02(10 \%)$ \\
2017 & $05(25 \%)$ \\
2018 & $02(10 \%)$ \\
\hline Total & $\mathbf{2 0}(\mathbf{1 0 0 \%})$ \\
\hline
\end{tabular}

Fonte: Autores.

Tabela 2. Distribuição dos estudos conforme programa.

\begin{tabular}{|c|c|}
\hline Programa & Frequência (\%) \\
\hline Enfermagem & $06(30 \%)$ \\
\hline Psicologia & $04(20 \%)$ \\
\hline Ciências da Saúde & $03(15 \%)$ \\
\hline Saúde Pública & $02(10 \%)$ \\
\hline Neuropsiquiatria & $01(5 \%)$ \\
\hline Educação & $01(5 \%)$ \\
\hline $\begin{array}{l}\text { Políticas sociais e } \\
\text { cidadania }\end{array}$ & $01(5 \%)$ \\
\hline Saúde da família & $01(5 \%)$ \\
\hline $\begin{array}{l}\text { Sociedade, cultura e } \\
\text { fronteira }\end{array}$ & $01(5 \%)$ \\
\hline Total & $20(100 \%)$ \\
\hline
\end{tabular}

Fonte: Autores.

Tabela 3. Distribuição dos estudos conforme região da instituição.

\begin{tabular}{cc}
\hline Região & Frequência (\%) \\
\hline Nordeste & $08(40 \%)$ \\
Sudeste & $08(40 \%)$ \\
Sul & $03(15 \%)$ \\
Centro-oeste & $01(5 \%)$ \\
Norte & $00(0 \%)$ \\
\hline Total & $\mathbf{2 0}(\mathbf{1 0 0 \%})$ \\
\hline
\end{tabular}

Fonte: Autores.

Iniciando a analise na Tabela 1, percebe-se que os estudos iniciaram a partir de 2005 até o ano de 2018, sendo que no ano de 2012 e 2015 não foram encontrados estudos. Conforme a Tabela 2 evidenciamos que programas de pós-graduação em enfermagem é o que mais produz estudos com essa temática com 9 (45\%) estudos, seguido de psicologia 3 (15\%), saúde pública 2(10\%), Neuropsiquiatria 1 (5\%), Educação 1(5\%) e Sociedade, Cultura e Fronteiras 1 (5\%). Quanto a distribuição conforme região geográfica (Tabela 3) dos estudos, 8 (40\%) foram produzidos na região Nordeste, 8 (40\%) na região Sudeste, $3(15 \%)$ na região Sul e $1(5 \%)$ na região Centro-Oeste, sendo a região Norte a única sem produções sobre o tema. 
Com relação a metodologia aplicada nos estudos, destaca-se que todos possuíam abordagem qualitativa. Os estudos qualitativos foram realizados principalmente utilizando entrevistas, ou entrevistas aliadas a outros métodos de coleta de dados, sendo que apenas 1 estudo não se utilizou de entrevistas em sua metodologia.

Após leitura dos estudos as temáticas que emergiram foram: O cuidado em saúde mental e a prática profissional na assistência em saúde mental.

\section{Discussão}

A enfermagem, enquanto ciência é percebida como recente, porém vista de maneira próspera, assim tem-se a pesquisa como ação que estabelece as fronteiras de uma disciplina, sendo fundamental a reflexão sobre as experiências e realizações da enfermagem, responsável então pelo crescimento e desenvolvimento do conhecimento desta (Guimarães et al, 2020).

A pós-graduação stricto sensu e latto sensu são recentes no Brasil quando comparada com outros níveis educacionais, estas são organizadas conforme a Lei no 9394/1996 a qual trata da Educação Superior (Filho et al, 2019). A produção encontrada nas teses e dissertações, disponíveis no portal da Coordenação de Aperfeiçoamento de Pessoal de Nível Superior (CAPES), traz um panorama das produções da pós-graduação brasileira. Desta forma a pesquisa nesse banco de dados é um importante mecanismo para o planejamento de futuros estudos na pós-graduação brasileira.

Conforme os estudos encontrados nessa pesquisa a defesa das produções acontece em um período que tem inicio no ano de 2005, e se distribuem de forma heterogênea até o ano de 2018, onde as produções variam de 0 até 5 por ano. O CAPS é um serviço que teve inicio em 1986, mas sua estruturação com relação aos profissionais atuantes se dá pela portaria ${ }^{\circ} 336$ em 2002, então é possível uma relação entre o marco temporal da legislação e o ano que foi observado o inicio da produção sobre o tema.

Com relação a localização geográfica dos estudos encontrados, estes se dão em sua maioria na região Nordeste e Sudeste, seguidos do Sul e Centro-oeste. Pode-se relacionar tais números com a cobertura do CAPS por região. Conforme os dados do relatório sobre saúde mental de 2014, a cobertura de CAPS por 100 mil habitantes apresenta o seguinte índice conforme região: Norte 0,61, Nordeste 1,0; Centro-oeste 0,66; Sudeste 0,79 e Sul 1,07, sendo a cobertura nacional 0,86. Desta forma os estudos são prevalentes nas regiões com maior cobertura de CAPS no Brasil.

A seguir serão discutidos os eixos temáticos que foram estabelecidos após análise dos estudos selecionados:

\section{O cuidado em saúde mental}

As necessidades em saúde de cada país estão estruturadas em sua população especifica, desse modo tem-se o fator político social como fundamental para compreender o contexto de saúde de uma população (Riveros, 2020). Entender a rede que compõe o cuidado do usuário é fundamental para pensar em como superar estigmas do sujeito com transtorno mental.

Na reinserção dos usuários do CAPS é observada uma vivencia de preconceito dentro da sociedade, fator histórico no desafio a constituição de seus direitos de cidadãos (E3, E6, E19). Há uma relação histórica entre questões de violência e pessoas com transtorno mental, descrevendo sujeitos de maneira perigosa e indesejada para convívio com outros gerando um processo de exclusão por seus sintomas (Monteiro \& Passos, 2019).

A dificuldade em articulações do cuidado com a rede é uma realidade no cotidiano da prática profissional dentro dos CAPS (E2, E5, E9), assim há uma percepção de atuação em uma rede frágil (E8). A questão humanitária do cuidado passa por estabelecer e reestabelecer a dignidade e a igualdade de direitos das pessoas em dificuldades, buscando seu acesso à política, sociedade e cultura. Assim, abrange-se um encontro com práticas profissionais éticas no que tange o cuidado em saúde (Riveros, 2020). Ainda que exista um esforço no processo de reinserção do usuário na sociedade (E6) é possível identificar um 
discurso dicotômico nos serviços, as práticas aparecem de forma que remetem a reforma psiquiátrica e em outros momentos se mostra voltada a um cuidado biomédico (E2, E4, E6, E13, E17).

Sob a lógica do cuidado ampliado, tem-se a Política Nacional de Humanização (PNH), que trata da produção de novos modos de cuidar e organizar o trabalho para uma melhor atenção em saúde. A PNH busca fortalecer as práticas de saúde e consolidar a Redes de Atenção Psicossocial (RAPS), pautada nos princípios da intersetorialidade, integralidade e interdisciplinaridade no cuidado (Brasil, 2013).

Pensando na interdisciplinaridade, essa é entendida como uma superação de pensamentos simplórios sobre os processos de saúde e doença, exigindo uma intensa interação entre especialidades para um cuidado mais qualificado com base na singularidade de casa pessoa (Brasil, 2013; Moreira, 2014). No discurso dos profissionais observa-se que há falta de interdisciplinaridades nas práticas, constituindo assim um cuidado sem corresponsabilização nas ações desenvolvidas para o cuidado do usuário (E15, E20).

Para além de um olhar sobre uma rede de saúde é essencial na integralidade do cuidado do sujeito assistido, articular cuidados envoltos em um reconhecimento do ser cidadão, então são importantes questões de sua vida social, como família, emprego, lazer e a autonomia do usuário (E3, E5, E17, E19). A intersetorialidade é importante nesse contexto, sendo que a partir desta é realizada uma ação além do campo da prática, alcançando políticas públicas (Silva et al., 2019).

As dificuldades vivenciadas por profissionais de saúde no cotidiano de trabalho compromete a efetivação dos princípios e diretrizes do SUS (Gontijo et. al, 2020). Uma prática frágil dentro do CAPS pode ser percebida no discurso dos profissionais, onde é apontada a carência de qualificação por meio de ações de educação permanente e ainda que seja reconhecida a importância desta (E2, E5, E6, E7, E8, E11, E13, E16, E18, E19). Nesse contexto, trabalhar em saúde mental é reconhecer as subjetividades do sujeito cuidado, buscando ajudar o usuário a significar suas vivências estabelecendo um relacionamento interpessoal terapêutico (Monteiro \& Passos, 2019; Morais, Pereira \& Silva, 2020).

As transformações necessárias nos modelos de atenção em saúde mental vão para além da implementação e ampliação das redes de serviços, elas conduzem a outro saber, que exige flexibilidade nas ações e papéis dos diversos profissionais, e pedem uma mudança individual e coletiva de todos os envolvidos no processo de reabilitação psicossocial. No cuidado em saúde mental, o CAPS tem como principal papel proporcionar a autonomia dos usuários, motivando-os ao convício social. (E1, E12, E14).

O cotidiano da pratica profissional do cuidado em saúde mental enfrenta dificuldades na articulação com a rede em saúde, evidenciando a não corresponsabilização das ações desenvolvidas pelos profissionais. O cuidado em saúde mental ainda presencia o preconceito da sociedade e a falta de interdisciplinaridades nas práticas.

\section{A prática profissional no Centro de Atenção Psicossocial}

Saúde e direitos humanos fazem parte da história de como se constituiu o cuidado em saúde, assim é importante reconhecer a relevância do cuidado prestado ao usuário do serviço sem esquecer o cuidado sobre o sujeito que presta assistência. É importante conhecer o que o trabalho significa para o profissional, assim por meio da práxis é possível identificar fatores positivos e negativos das vivências (Silva et al, 2019).

Desde o final dos anos 80 a violência no ambiente de trabalho tem sido apontada como risco ocupacional e responsável pelo afastamento de profissionais de seu ambiente de serviço, esta é considerada como qualquer ação individual ou grupo que cause danos físicos ou psicológicos (Monteiro \& Passos, 2019). O cuidar de si é pautado em uma prática éticopolitica, remetendo a questões filosóficas sobre o lugar do sujeito no mundo, as quais conforme reflexão se aproxima de algo que pode ser chamado de felicidade ou harmonia (Rohden \& Kussler, 2017). 
Conforme preconizado na Reforma Psiquiátrica brasileira, o cuidado do sujeito com transtorno mental deve ser de base territorial e valorizando suas subjetividades, assim o CAPS deve ser um serviço que se articule com o território onde a pessoa reside. Contudo, ainda são observadas nos CAPS, práticas que remetem a um cuidado biomédico, com saberes voltados para a prática do médico e valorização de um cuidado dentro do serviço (E2, E6, E17).

As práticas dentro do CAPS são constituídas algumas vezes sem qualificação, ou seja, são baseadas na vivência da prática do próprio profissional dentro do serviço (E16, E18). Este pode ser um fator que colabore ao fato de no cuidado existirem profissionais que trazem percepções pessoais no manejo, o que pode constituir prejulgamento sobre o sujeito que está sendo cuidado (E4, E8, E10).

No ambiente de trabalho o profissional necessita de equilíbrio emocional. Contudo, o contexto brasileiro expõe o trabalhador à falta de investimentos o que torna as condições de trabalho precárias, contribuindo ao adoecimento do profissional e comprometendo a qualidade do cuidado prestado no serviço de saúde (Lourenção, 2018).

Em relação ao sofrimento do profissional é importante que exista uma analise subjetiva, na qual seja possível observar o fenômeno vivenciado pelo sujeito com sensibilidade e compreensibilidade. É importante que investigadores, gestores e coordenadores entendam esse processo de sofrimento e construam estratégias de enfrentamento para melhorar a qualidade do ambiente de serviço dos profissionais (Schaefer, Zoboli \& Vieira, 2018).

Observa-se a existência de profissionais frustrados por não conseguirem desempenhar suas funções conforme propostas preconizadas para seu ambiente de trabalho (Gontijo et al, 2020). A partir da realidade enfrentada no cotidiano de prática dos profissionais, partindo do que é observado de resultados no tratamento dos usuários, há o questionamento sobre o quanto transformadora é a prática dentro do CAPS (E7).

A exaustão e despersonalização são apontadas como fatores de risco ao surgimento de um caso de sofrimento psíquico em profissionais. Essas causam diminuição ou perda de recursos emocionais, atitudes negativas em relação aos usuários, sentimento de incompetência e diminuição da percepção de realização no trabalho (Cruz et al, 2019).

Entendendo o cuidar como uma arte, podem-se relacionar resultados do cuidado ao usuário por meio de uma prática que se constitui na relação entre usuário e ambiente, profissional e usuário e profissional e ambiente (Riveros, 2020). Para um trabalho que contemple as necessidades do usuário do CAPS de maneira integral passa por uma construção multiprofissional onde a comunicação entre profissionais é essencial (E7, E11). Um cuidado humanizado passa por práticas onde a comunicação é importante a partir de uma aproximação com o real (Riveros, 2020), ou seja, quando se está em um ato concreto de cuidado se torna fundamental uma comunicação adequada.

A prática profissional, por vezes, é baseada na vivência do próprio profissional dentro do serviço sem uma devida qualificação. Observa-se a prática no contexto dos CAPS, ainda pautada a um cuidado biomédico com a valorização de um saber uniprofissional.

\section{Considerações Finais}

Por meio deste estudo foi possível caracterizar produções científicas dos programas de pós-graduação de mestrado e doutorado acerca da prática profissional em CAPS. As pesquisas qualitativas foram predominantemente utilizadas, estas dão voz aos sujeitos envolvidos na prática profissional, possibilitando que expressem de maneira objetiva e subjetiva suas vivencias e iniciem um processo de empoderamento sobre sua prática por meio da reflexão e crítica.

Destaca-se a importância da realização de estudos que tragam um debate sobre a realidade vivenciada no cotidiano dos profissionais, incentivando pesquisas que qualifiquem a prática dos profissionais dentro dos CAPS e na saúde mental de modo geral. A existência de pesquisas ao longo dos anos com a temática de profissionais e CAPS demonstra uma preocupação com o cuidado prestado nesses serviços, indo ao encontro dos pressupostos da Reforma Psiquiatra e de uma ampla, há uma 
preocupação além dos usuários, mas com os profissionais envolvidos no cuidado.

\section{Referências}

Bardin, L. Análise de Conteúdo. Edições 70 LDA.

Carvalho, M. D. F. A. A., Coelho, E. D. A. C., Oliveira, J. F. D., Araújo, R. T. D., \& Barros, A. R. (2017). Desarticulação da rede psicossocial comprometendo a integralidade do cuidado. Revista da Escola de Enfermagem da USP, 51.

Brasil. Lei $n^{\circ}$ 10216. Casa civil. Subchefia de assuntos jurídicos.

Brasil. Ministério da Saúde. Portaria 336.

Brasil. Ministério da Saúde. Portaria nº3088.

Brasil. Ministério da Saúde. Política Nacional de Humanização - PNH.

Brasil. Ministério da saúde. Saúde Mental em Dados 12. .

Cruz, S. P. D. L., Cruz, J. C., Cabrera, J. H., \& Abellán, M. V. (2019). Fatores relacionados à probabilidade de sofrer problemas de saúde mental em profissionais de emergência. Revista Latino-Americana de Enfermagem, 27.

Dias, M. O., Souza, N. V. D. D. O., Penna, L. H. G., \& Gallasch, C. H. (2019). Percepção das lideranças de enfermagem sobre a luta contra a precarização das condições de trabalho. Revista da Escola de Enfermagem da USP, 53.

Ferreira, J. T., Mesquita, N. N. M., Silva, T. D., Silva, V. D., Lucas, W. J., \& Batista, E. C. (2016). Os Centros de Atenção Psicossocial (CAPS): uma instituição de referência no atendimento à saúde mental. Rev. Saberes, Rolim de Moura, 4(1), 72-86.

Viana Filho, M. V. C., Matos, T. G. R., Galindo, M. C. T., Silva, R. D., \& Vale, S. F. D. (2019). O trabalho do professor na pós-graduação no Brasil após a Lei No 9394/1996. Avaliação: Revista da Avaliação da Educação Superior (Campinas), 24(1), 127-147.

Foucault, M. A história da loucura. Editora Perspectiva. Edição: 11, 2019.

Gontijo, M. D., Viegas, S. M. D. F., Freitas, A. T. S., Maia, A. F. D. F., Nitschke, R. G., \& Nabarro, M. (2020). Atuação cotidiana no Sistema Único de Saúde em sua terceira década. Escola Anna Nery, 24(4).

Guimarães, G. D. L., Mendoza, I. Y. Q., Corrêa, A. D. R., Ribeiro, E. G., Guimarães, M. O., \& Chianca, T. C. M. (2020). A proposed evaluation of postgraduate nursing according to Thomas Kuhn. Texto \& Contexto-Enfermagem, 29.

Lourenção, L. G. (2018). Qualidade de vida, engagement, ansiedade e depressão entre gestores de Unidades da Atenção Primária à Saúde. Rev. port. enferm. saúde mental, 58-64.

Monteiro, C., \& Passos, J. (2019). A violência e os profissionais de saúde no hospital psiquiátrico. Revista Portuguesa de Enfermagem de Saúde Mental, (21), 54-61.

de Moraes, P. L. M., Pereira, E. R., \& Silva, R. M. C. R. A. (2020). A percepção da desinstitucionalização pelos profissionais da saúde mental na práxis no Centro de Atenção Psicossocial no contexto brasileiro. Research, Society and Development, 9(7), e763974750-e763974750.

Moreira, K. B. (2014). Contribuições para o aprimoramento dos processos de trabalho e cuidado no CAPS II: Fenomenologia existencial e Política Nacional de Humanização. Revista de Psicologia da UNESP, 13(2), 1-13.

Cruz Riveros, C. (2020). A natureza do cuidado humanizado. Enfermagem: Atendimento Humanizado, 9(1), 21-32.

Rohden, L., \& Kussler, L. M. (2017). Filosofar enquanto cuidado de si mesmo: um exercício espiritual ético-político. Trans/Form/Ação, 40(3), 93-112.

Santos, J. M. D., Baptista, J. Á., Nasi, C., \& Camatta, M. W. (2018). Responsabilização e participação: como superar o caráter tutelar no centro de atenção psicossocial álcool drogas? Revista Gaúcha de Enfermagem, 39.

Schaefer, R., Zoboli, E. L. C. P., \& Vieira, M. (2018). Sofrimento moral em enfermeiros: descrição do risco para profissionais. Texto \& ContextoEnfermagem, 27(4).

Silva, M. A., Teixeira, E. R., Pereira, E. R., Silva, R. M. C. R. A., Rocha, R. C. N. P., \& Rondon, S. O. V. (2019). Saúde como direito e cuidado de si: concepção dos profissionais de enfermagem. Revista Brasileira de Enfermagem, 72, 159-165.

Souza, H. A., \& Bernardo, M. H. (2019). Prevenção de adoecimento mental relacionado ao trabalho: a práxis de profissionais do Sistema Único de Saúde comprometidos com a saúde do trabalhador. Revista Brasileira de Saúde Ocupacional, 44. 